\title{
Time-Varying FIR Equalization for MIMO Transmission over Doubly Selective Channels
}

\author{
Imad Barhumi $^{1}$ and Marc Moonen (EURASIP Member) ${ }^{2}$ \\ ${ }^{1}$ College of Engineering, United Arab Emirates University (UAE), P.O. Box 17555 Al-Ain, United Arab Emirates \\ ${ }^{2}$ ESAT/SCD-KULeuven, Kasteelpark Arenberg 10, 3001 Leuven, Belgium
}

Correspondence should be addressed to Imad Barhumi, imad.barhumi@uaeu.ac.ae

Received 2 November 2009; Revised 13 March 2010; Accepted 8 April 2010

Academic Editor: Xiaoli Ma

Copyright ( 2010 I. Barhumi and M. Moonen. This is an open access article distributed under the Creative Commons Attribution License, which permits unrestricted use, distribution, and reproduction in any medium, provided the original work is properly cited.

\begin{abstract}
We propose time-varying FIR equalization techniques for spatial multiplexing-based multiple-input multiple-output (MIMO) transmission over doubly selective channels. The doubly selective channel is approximated using the basis expansion model (BEM), and equalized by means of time-varying FIR filters designed according to the BEM. By doing so, the time-varying deconvolution problem is converted into a two-dimensional time-invariant deconvolution problem in the time-invariant coefficients of the channel BEM and the time-invariant coefficients of the equalizer BEM. The timevarying FIR equalizers are derived based on either the matched filtering criterion, or the linear minimum mean-square error (MMSE) or the zero-forcing (ZF) criteria. In addition to the linear equalizers, the decision feedback equalizer (DFE) is proposed. The DFE can be designed according to two different scenarios. In the first scenario, the DFE is based on feeding back previously estimated symbols from one particular antenna at a time. Whereas, in the second scenario, the previously estimated symbols from all transmit antennas are fed back together. The performance of the proposed equalizers in the context of MIMO transmission is analyzed in terms of numerical simulations.
\end{abstract}

\section{Introduction}

The wireless communication industry has experienced a rapid growth in recent years, and digital cellular systems are currently designed to provide high data rates at high terminal speeds. High data rates give rise to intersymbol interference (ISI) due to multipath fading. Such ISI channels are described as frequency-selective. On the other hand, due to the user's mobility and/or receiver carrier frequency offset (CFO) the received signal is subject to frequency shifts. CFO in conjunction with the Doppler shift give rise to time-selectivity characteristics of the mobile wireless channel. Therefore, the mobile wireless communication channel is generally characterized as time- and frequency selective channel or the so-called doubly selective, which in turns causes degradation in the system performance. This motivates the search for efficient and robust equalization techniques to improve on the information transmission reliability.
The data rate provided by the wireless communication system can be increased substantially by using multiple antennas at the transmitter and at the receiver. It is well-known that multiple-input multiple-output (MIMO) systems can provide an increase in the system capacity by a factor linear in the minimum number of the antennas used at either the transmitter or the receiver $[1,2]$. In this paper, we address the problem of equalization for spatial multiplexing-based MIMO transmission over doubly selective channels. Linear and nonlinear decision feedback equalizers are proposed based on the minimum mean-square error (MMSE) and the zero-forcing (ZF) criteria.

For slowly time-varying channel, adaptive techniques for channel estimation or equalization are developed to combat the problem of ISI. These algorithms range from the least mean-squares (LMS) algorithm [3], to the recursive least squares (RLS) algorithm or Kalman filtering algorithm [4]. For fast flat-fading channels, polynomial fitting of the 1tap time-varying channel is used to predict the channel 
as proposed in [5]. Extending the polynomial fitting over the whole packet (or using a sliding window approach) to time-varying frequency-selective channels is investigated in [6]. For single-input multiple-output (SIMO) transmission over doubly selective channels, ZF and MMSE time-varying FIR equalization techniques were proposed in [7]. The extension to the DFE equalizer was presented in [8]. In the MIMO context, the authors in [9] propose block linear filters to mitigate intercarrier interference (ICI) for OFDM transmission over time-varying multipath fading channels. A Kalman filter based MMSE interference suppression is proposed in [10] for MIMO transmission over doubly selective channels. In there, a two-stage suppression technique is proposed; one stage is used to mitigate ISI due to channel frequency selectivity, and another stage is used to mitigate ICI due to channel time selectivity. For estimation of MIMO doubly selective channels, an MMSE pilot-aided transmission is proposed in [11] for cyclic prefix (CP) based block transmission scheme. Optimal training design is proposed in [12]. Adaptive estimation of doubly selective channels is proposed in [13]. There in a subblock tracking scheme for the basis expansion model (BEM) coefficients of the doubly selective channel using periodically transmitted training symbols.

In this paper, we propose matched filter (MF), ZF, MMSE, and DFE time-varying FIR equalizers for MIMO transmission over doubly selective channels. Spatial multiplexing, where independent data streams are assumed on different transmit antennas, is considered for the MIMO transmission. Considering other MIMO transmission techniques, for example, space-time block coding (STBC) [14] is out of the scope of this paper. In the above mentioned schemes, perfect channel state information (CSI) is assumed to be known at the receiver. The basis expansion model (BEM) $[15,16]$ is used to approximate the underlying communication channel, and to model and design the equalizers. In this sense, a large complex 1D time-varying deconvolution problem is turned into a lower complexity $2 \mathrm{D}$ deconvolution problem in the BEM coefficients of the channel and the BEM coefficients of the equalizers.

This paper is organized as follows. In Section 2, the system model is introduced. The time-varying FIR linear equalizers are developed in Section 3. The time-varying FIR DFEs are investigated in Section 4. Our findings are confirmed by numerical simulations introduced in Section 5 . Finally, conclusions are drawn in Section 6.

Notation. We use upper bold face letters and lower bold face letters to denote matrices and vectors, respectively. Superscripts ${ }^{H},{ }^{T}$, and $*$ represent Hermitian, transpose, and conjugate, respectively. To simplify notations and save space, the double summation over the subscripts $i$ and $j$ is denoted as $\sum_{i, j}$, where the ranges of $i$ and $j$ should be clear from the context. We denote the $N \times N$ identity matrix as $\mathbf{I}_{N}$, the $M \times N$ all-zero matrix as $\mathbf{0}_{M \times N}$. Finally, $\otimes$ denotes Kronecker product, $\oplus$ denotes the direct sum, and $\operatorname{diag}\{\mathbf{x}\}$ denotes the diagonal matrix with vector $\mathbf{x}$ on its diagonal.

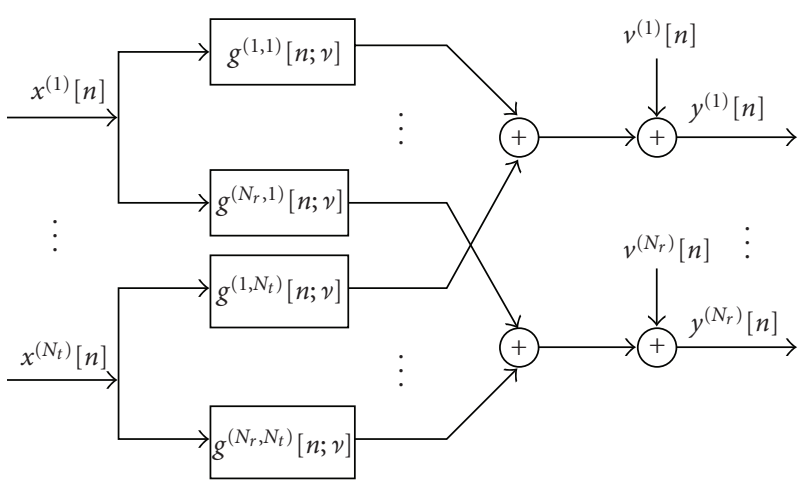

Figure 1: System model.

\section{System Model}

We consider an MIMO system with $N_{t}$ transmit antennas and $N_{r}$ receive antennas. The input data stream is spatially multiplexed across the $N_{t}$ transmit antennas, and transmitted over the time-varying multipath fading channel at a rate of $1 / T$ symbols/s. Assuming that $\tau_{\max }$ is the maximum delay spread of all channels and the received symbols are sampled at $1 / T$ samples/s, the channel order $L$ is then obtained as $L=\left\lfloor\tau_{\max } / T\right\rfloor+1$. The $l$ th tap of the time-varying channel characterizing the link between the $t$ th transmit antenna and the $r$ th receive antenna at time-index $n$ is denoted as $g^{(r, t)}[n ; l]$ for $l=0, \ldots, L$. The baseband received signal at the $r$ th receive antenna at time-index $n$ is obtained as

$$
y^{(r)}[n]=\sum_{t=1}^{N_{t}} \sum_{l=0}^{L} g^{(r, t)}[n ; l] x^{(t)}[n-l]+v^{(r)}[n],
$$

where $x^{(t)}[n]$ is the QAM symbol transmitted from the $t$ th antenna at time-index $n$, and $v^{(r)}[n]$ is the additive noise at the $r$ th receive antenna. The baseband equivalent of the system described by (1) is depicted in Figure 1.

We will use the BEM [15-17] to approximate the doubly selective channel $g^{(r, t)}[n ; l]$ for $n \in\left\{0, \ldots, N+L^{\prime}-1\right\}$ ( $L^{\prime}$ will be the time-varying FIR equalizer order). In this model, the channel is characterized as a time-varying FIR filter, where each tap of the time-varying FIR is expressed as a superposition of time-varying complex exponential basis functions with frequencies on a DFT grid. The lth tap of the approximated time-varying FIR channel between the $t$ th transmit antenna and the $r$ th receive antenna at time-index $n$ is expressed as

$$
h^{(r, t)}[n ; l]=\sum_{q=-Q / 2}^{Q / 2} h_{q, l}^{(r, t)} e^{j 2 \pi q n / K},
$$

where $Q$ is the number of time-varying basis functions. Suppose $f_{\max }$ is the maximum frequency offset of all channels, the number of time-varying basis functions is chosen such that it satisfies $Q /(2 K T) \geq f_{\max }$, where $K$ is the BEM resolution. In general $K$ is chosen as an integer multiple of the Block size $N$. The coefficients $h_{q, l}^{(r, t)}$ are kept invariant over a block of $N+L^{\prime}$ symbols. Substituting the 


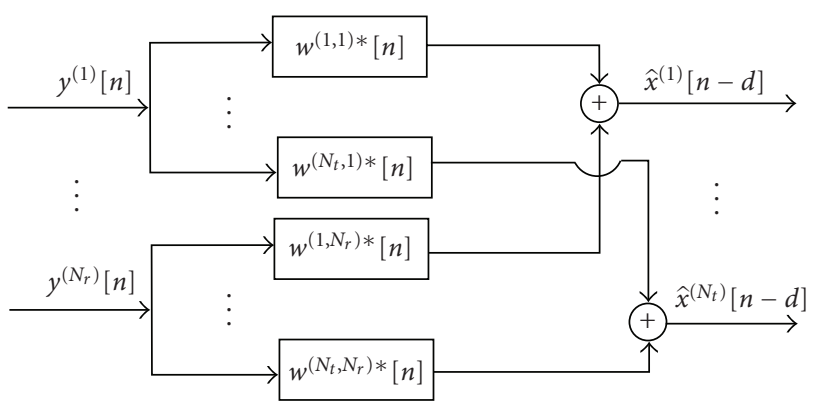

FIgure 2: Time-varying FIR linear equalization for MIMO transmission.

channel BEM (2) into (1) leads to the following: (note that we use different notations for the true channel $g[n ; l]$ and the BEM channel $h[n ; l]$ to stress the fact that the BEM model is an approximation of the true channel. However, in the subsequent analysis we proceed as if the channel follows exactly the BEM)

$$
y^{(r)}[n]=\sum_{t=1}^{N_{t}} \sum_{q, l} e^{j 2 \pi q n / K} h_{q, l}^{(r, t)} x^{(t)}[n-l]+v^{(r)}[n] .
$$

Casting the received symbols into blocks of length $N+L^{\prime}$, the received vector at the $r$ th receive antenna is obtained as

$$
\mathbf{y}^{(r)}=\sum_{t=1}^{N_{t}} \sum_{q, l} h_{q, l}^{(r, t)} \mathbf{D}_{q} \mathbf{Z}_{l} \mathbf{x}^{(t)}+\mathbf{v}^{(r)}
$$

where $\mathbf{y}^{(r)}=\left[y^{(r)}\left[-L^{\prime}\right], \ldots, y^{(r)}[N-1]\right]^{T}, \mathbf{x}^{(t)}=\left[x^{(t)}[-L-\right.$ $\left.\left.L^{\prime}\right], \ldots, x^{(t)}[N-1]\right]^{T}$, and $\mathbf{v}^{(r)}$ is similarly defined as $\mathbf{y}^{(r)}$. The diagonal matrix $\mathbf{D}_{q}$ representing the $q$ th basis function is defined as $\mathbf{D}_{q}=\operatorname{diag}\left\{\left[1, \ldots, e^{j 2 \pi q\left(N+L^{\prime}-1\right) / K}\right]^{T}\right\}$, and the $\left(N+L^{\prime}\right) \times\left(N+L^{\prime}+L\right)$ Toeplitz matrix $\mathbf{Z}_{l}$ is defined as $\mathbf{Z}_{l}=\left[\mathbf{0}_{N+L^{\prime} \times(L-l)}, \mathbf{I}_{N+L^{\prime}}, \mathbf{0}_{\left(N+L^{\prime}\right) \times l}\right]$.

\section{Linear Time-Varying FIR Equalization}

In this section, linear time-varying FIR equalizers are derived based on matched filtering (MF), MMSE, and ZF criteria. Each of these linear equalizers consists of a bank of $N_{t}$ time-varying FIR filters applied to each receive antenna. The outputs are combined as shown in Figure 2 to estimate the transmitted symbols of all data streams on all transmit antennas. An estimate of the symbol of the ath transmit antenna at time-index $n$ subject to some decision delay $d$ can be obtained as

$$
\hat{x}^{(a)}[n-d]=\sum_{r=1}^{N_{r}} \sum_{l^{\prime}=0}^{L^{\prime}} w^{(a, r) *}\left[n ; l^{\prime}\right] y^{(r)}\left[n-l^{\prime}\right],
$$

where $w^{(a, r)}\left[n ; l^{\prime}\right]$ is the time-varying FIR equalizer corresponding to the ath transmit antenna and the $r$ th receive antenna, and $L^{\prime}$ is the order of all filters. As the channel is approximated using the BEM, the BEM can also be used to model the time-varying FIR equalizers. The time variation of each tap is then composed of a superposition of $Q^{\prime}+1$ complex exponential basis functions with frequencies on the same DFT grid as the BEM of the time-varying channel. Therefore, the $l^{\prime}$ th tap of the time-varying FIR equalizer $w^{(a, r)}\left[n ; l^{\prime}\right]$ at time-index $n$ for $n \in\{0, \ldots, N-1\}$ is modeled as

$$
w^{(a, r)}\left[n ; l^{\prime}\right]=\sum_{q^{\prime}=-Q^{\prime} / 2}^{Q^{\prime} / 2} w_{q^{\prime}, l^{\prime}}^{(a, r)} e^{-j 2 \pi q^{\prime} n / K},
$$

where $w_{q^{\prime}, l^{\prime}}^{(a, r)}$ is the BEM coefficient of the $q^{\prime}$ th basis of $w^{(a, r)}\left[n ; l^{\prime}\right]$. Substituting (6) into (5) an estimate of the transmitted symbol on the ath antenna at time-index $n$ can be obtained as

$$
\hat{x}^{(a)}[n-d]=\sum_{r=1}^{N_{r}} \sum_{q^{\prime}, l^{\prime}} e^{j 2 \pi q^{\prime} n / K} w_{q^{\prime}, l^{\prime}}^{(a, r) *} y^{(r)}\left[n-l^{\prime}\right] .
$$

On a block level formulation (vector-matrix form), (7) can be written as

$$
\hat{x}^{(a)}=\sum_{r=1}^{N_{r}} \sum_{q^{\prime}, l^{\prime}} w_{q^{\prime}, l^{\prime}}^{(a, r) *} \overline{\mathbf{D}}_{q^{\prime}} \overline{\mathbf{Z}}_{l^{\prime}} \mathbf{y}^{(r)},
$$

where the vector of the estimated symbols $\hat{\mathbf{x}}^{(a)}=$ $\left[\hat{x}^{(a)}[-d], \ldots, \hat{x}^{(a)}[N-d+1]\right]^{T}$, the $N \times N$ diagonal matrix $\overline{\mathbf{D}}_{q^{\prime}}=\operatorname{diag}\left\{\left[1, \ldots, e^{j 2 \pi q^{\prime}(N-1) / K}\right]^{T}\right\}$, and the $N \times\left(N+L^{\prime}\right)$ Toeplitz matrix $\overline{\mathbf{Z}}_{l^{\prime}}=\left[\mathbf{0}_{N \times\left(L^{\prime}-l^{\prime}\right)}, \mathbf{I}_{N}, \mathbf{0}_{N \times l^{\prime}}\right]$. Substituting (4) into (8) yields

$$
\begin{aligned}
\hat{\mathbf{x}}^{(a)}= & \sum_{r=1}^{N_{r}} \sum_{t=1}^{N_{t}} \sum_{q^{\prime}, l^{\prime}} \sum_{q, l} w_{q^{\prime}, l^{\prime}}^{(a, r) *} h_{q, l}^{(r, t)} \overline{\mathbf{D}}_{q^{\prime}} \overline{\mathbf{Z}}_{l^{\prime}} \mathbf{D}_{q} \mathbf{Z}_{l} \mathbf{x}^{(t)} \\
& +\sum_{r=1}^{N_{r}} \sum_{q^{\prime}, l^{\prime}} w_{q^{\prime}, l^{\prime}}^{(a, r) *} \overline{\mathbf{D}}_{q^{\prime}} \overline{\mathbf{Z}}_{l^{\prime}} \mathbf{v}^{(r)} .
\end{aligned}
$$

The formula (9) can be further simplified using the property $\overline{\mathbf{Z}}_{l^{\prime}} \mathbf{D}_{q}=e^{j 2 \pi q\left(L^{\prime}-l^{\prime}\right) / K} \overline{\mathbf{D}}_{q} \overline{\mathbf{Z}}_{l^{\prime}}$, which leads to

$$
\begin{aligned}
\hat{\mathbf{x}}^{(a)}= & \sum_{r=1}^{N_{r}} \sum_{t=1}^{N_{t}} \sum_{q^{\prime}, l^{\prime}} \sum_{q, l} e^{j 2 \pi q\left(L^{\prime}-l^{\prime}\right) / K} w_{q^{\prime}, l^{\prime}}^{(a, r) *} h_{q, l}^{(r, t)} \overline{\mathbf{D}}_{p} \widetilde{\mathbf{Z}}_{k} \mathbf{x}^{(t)} \\
& +\sum_{r=1}^{N_{r}} \sum_{q^{\prime}, l^{\prime}} w_{q^{\prime}, l^{\prime}}^{(a, r) *} \overline{\mathbf{D}}_{q^{\prime}} \overline{\mathbf{Z}}_{l^{\prime}} \mathbf{v}^{(r)},
\end{aligned}
$$

where $p=q+q^{\prime}, k=l+l^{\prime}$, and the $N \times\left(N+L+L^{\prime}\right)$ matrix $\widetilde{\mathbf{Z}}_{k}=\left[\mathbf{0}_{N \times\left(L+L^{\prime}-k\right)}, \mathbf{I}_{N}, \mathbf{0}_{N \times k}\right]$. We can now define $f_{p, k}^{(a, t)}$ as

$$
f_{p, k}^{(a, t)}=\sum_{r=1}^{N_{r}} \sum_{q^{\prime}, l^{\prime}} e^{j 2 \pi\left(p-q^{\prime}\right)\left(L^{\prime}-l^{\prime}\right) / K} w_{q^{\prime}, l^{\prime}}^{(a, r) *} h_{p-q^{\prime}, k-l^{\prime}}^{(r, t)},
$$

which is a $2 \mathrm{D}$ function representing a weighted $2 \mathrm{D}$ convolution in the time-invariant BEM coefficients of the equalizer and the time-invariant BEM coefficients of the channel. Substituting (11) into (10) yields

$$
\widehat{\mathbf{x}}^{(a)}=\sum_{t=1}^{N_{t}} \sum_{p, k} f_{p, k}^{(a, t)} \overline{\mathbf{D}}_{p} \widetilde{\mathbf{Z}}_{k} \mathbf{x}^{(t)}+\sum_{r=1}^{N_{r}} \sum_{q^{\prime}, l^{\prime}} w_{q^{\prime}, l^{\prime}}^{(a, r) *} \overline{\mathbf{D}}_{q^{\prime}} \overline{\mathbf{Z}}_{l^{\prime}} \mathbf{v}^{(r)} \text {. }
$$


Defining $\mathbf{f}^{(a, t)}=\left[f_{-\left(Q+Q^{\prime}\right) / 2,0}^{(a, t)}, \ldots, f_{-\left(Q+Q^{\prime}\right) / 2, L+L^{\prime}}^{(a, t)}, \ldots\right.$, $\left.f_{\left(Q+Q^{\prime}\right) / 2, L+L^{\prime}}^{(a, t)}\right]^{T}$ and $\mathbf{f}^{(a)}=\left[\mathbf{f}^{(a, 1) T}, \ldots, \mathbf{f}^{\left(a, N_{t}\right) T}\right]^{T}$, (12) can finally be written as

$$
\hat{\mathbf{x}}^{(a)}=\left(\mathbf{f}^{(a) T} \otimes \mathbf{I}_{N}\right)\left(\mathbf{I}_{N_{t}} \otimes \mathbf{A}\right) \mathbf{x}+\left(\mathbf{w}^{(a) H} \otimes \mathbf{I}_{N}\right)\left(\mathbf{I}_{N_{r}} \otimes \mathbf{B}\right) \mathbf{v}
$$

where $\mathbf{x}=\left[\mathbf{x}^{(1) T}, \ldots, \mathbf{x}^{\left(N_{t}\right) T}\right]^{T}, \mathbf{v}=\left[\mathbf{v}^{(1) T}, \ldots, \mathbf{v}^{\left(N_{r}\right) T}\right]^{T}$, and the matrices $\mathbf{A}$ and $\mathbf{B}$ are defined as

$$
\mathbf{A}=\left[\begin{array}{c}
\overline{\mathbf{D}}_{-\left(Q+Q^{\prime}\right) / 2} \tilde{\mathbf{Z}}_{0} \\
\vdots \\
\overline{\mathbf{D}}_{-\left(Q+Q^{\prime}\right) / 2} \tilde{\mathbf{Z}}_{L+L^{\prime}} \\
\vdots \\
\overline{\mathbf{D}}_{\left(Q+Q^{\prime}\right) / 2} \tilde{\mathbf{Z}}_{L+L^{\prime}}
\end{array}\right], \quad \mathbf{B}=\left[\begin{array}{c}
\overline{\mathbf{D}}_{-Q^{\prime} / 2} \overline{\mathbf{Z}}_{0} \\
\vdots \\
\overline{\mathbf{D}}_{-Q^{\prime} / 2} \overline{\mathbf{Z}}_{L^{\prime}} \\
\vdots \\
\overline{\mathbf{D}}_{Q^{\prime} / 2} \overline{\mathbf{Z}}_{L^{\prime}}
\end{array}\right]
$$

The time-varying FIR equalizer BEM coefficients vector is defined as $\mathbf{w}^{(a)}=\left[\mathbf{w}^{(a, 1) T}, \ldots, \mathbf{w}^{\left(a, N_{r}\right) T}\right]^{T}$ with $\mathbf{w}^{(a, r)}=$ $\left[w_{-Q^{\prime} / 2,0}^{(a, r)}, \ldots, w_{-Q^{\prime} / 2, L^{\prime}}^{(a, r)} \ldots, w_{Q^{\prime} / 2, L^{\prime}}^{(a, r)}\right]^{T}$. We can derive a relationship between $\mathbf{w}^{(a)}$ and $\mathbf{f}^{(a)}$ as follows. First, define the $\left(L^{\prime}+1\right) \times\left(L+L^{\prime}+1\right)$ block Toeplitz matrix $\mathscr{H}_{q}^{(r, t)}$ as

$$
\boldsymbol{H}_{q}^{(r, t)}=\left[\begin{array}{cccc}
h_{q, 0}^{(r, t)} & \ldots & h_{q, L}^{(r, t)} & \mathbf{0} \\
& \ddots & \ddots & \\
\mathbf{0} & h_{q, 0}^{(r, t)} & \ldots & h_{q, L}^{(r, t)}
\end{array}\right],
$$

then define the $\left(Q^{\prime}+1\right)\left(L^{\prime}+1\right) \times\left(Q+Q^{\prime}+1\right)\left(L+L^{\prime}+1\right)$ block Toeplitz matrix $\mathscr{H}^{(r, t)}$ as

$$
\begin{aligned}
& \mathscr{H}^{(r, t)} \\
& =\left[\begin{array}{cccc}
\boldsymbol{\Omega}_{-Q / 2} \mathscr{H}_{-Q / 2}^{(r, t)} & \ldots & \boldsymbol{\Omega}_{Q / 2} \boldsymbol{H}_{Q / 2}^{(r, t)} & \\
\mathbf{0} & \ddots & \ddots & \mathbf{0} \\
& \boldsymbol{\Omega}_{-Q / 2} \mathscr{H}_{-Q / 2}^{(r, t)} & \ldots & \boldsymbol{\Omega}_{Q / 2} \boldsymbol{H}_{Q / 2}^{(r, t)}
\end{array}\right],
\end{aligned}
$$

with $\boldsymbol{\Omega}_{q}=\operatorname{diag}\left\{\left[e^{j 2 \pi q L^{\prime} / K}, \ldots, 1\right]^{T}\right\}$. Defining $\mathscr{H}^{(t)}=$ $\left[\mathscr{H}^{(1, t) T}, \ldots, \mathscr{H}^{\left(N_{r}, t\right) T}\right]^{T}$ and $\boldsymbol{H}=\left[\mathscr{H}^{(1)}, \ldots, \mathscr{H}^{\left(N_{t}\right)}\right]$, we can derive from (11) the following relationship in the BEM coefficients of the time-varying FIR channel and the BEM coefficients of the time-varying FIR equalizers as

$$
\mathbf{f}^{(a) T}=\mathbf{w}^{(a) H} \mathscr{H} .
$$

3.1. Matched Filter Equalizer. The matched filter (MF) equalizer is the optimal linear equalizer (filter) that maximizes the signal-to-noise ratio (SNR) without necessarily canceling ISI. Defining the $\mathrm{SNR}_{o}$ to be the ratio of the power of the MF output due to the desired signal to the power of the MF output due to the noise as

$$
\mathrm{SNR}_{o}=\frac{\mathrm{E}\left\{\mathbf{q}^{H} \mathbf{q}\right\}}{\mathrm{E}\left\{\mathbf{n}^{H} \mathbf{n}\right\}}=\frac{\operatorname{tr}\left\{\mathrm{E}\left\{\mathbf{q q}^{H}\right\}\right\}}{\operatorname{tr}\left\{\mathrm{E}\left\{\mathbf{n} \mathbf{n}^{H}\right\}\right\}}
$$

where $\mathbf{q}$ and $\mathbf{n}$ are the first and the second terms of (13), respectively. In this definition, $\mathbf{q}$ constitutes the output due to the desired signal, while $\mathbf{n}$ constitutes the output due to noise. Before we proceed, let us first introduce the following properties:

$$
\begin{gathered}
\operatorname{tr}\left\{\left(\mathbf{a}^{T} \otimes \mathbf{I}_{N}\right) \mathbf{V}\right\}=\mathbf{a}^{T} \operatorname{subtr}_{N}\{\mathbf{V}\}, \\
\operatorname{tr}\left\{\left(\mathbf{a}^{T} \otimes \mathbf{I}_{N}\right) \mathbf{U}\left(\mathbf{a}^{*} \otimes \mathbf{I}_{N}\right)\right\}=\mathbf{a}^{T} \operatorname{subtr}_{N}\{\mathbf{U}\} \mathbf{a}^{*},
\end{gathered}
$$

for an arbitrary $k \times 1$ vector a, an arbitrary $k N \times N$ matrix $\mathbf{V}$, and an arbitrary $k N \times k N$ matrix $\mathbf{U}$. The operator $\operatorname{subtr}_{N}\{\cdot\}$ splits the matrix into $N \times N$ submatrices and replaces each submatrix by its trace (let $\mathbf{A}$ be a $p N \times q N$ matrix $\mathbf{A}=$ $\left[\begin{array}{ccc}\mathbf{A}_{11} & \ldots & \mathbf{A}_{1 q} \\ \vdots & \ddots & \vdots \\ \mathbf{A}_{p 1} & \cdots & \mathbf{A}_{p q}\end{array}\right]$, where $\mathbf{A}_{i j}$ is the $(i, j)$ th $N \times N$ submatrix of $\mathbf{A}$. The $p \times q$ matrix $\operatorname{subtr}_{N}\{\mathbf{A}\}$ is then defined as $\operatorname{subtr}_{N}\{\mathbf{A}\}=$ $\left[\begin{array}{llll}\operatorname{tr}\left\{\mathbf{A}_{11}\right\} & \ldots & \operatorname{tr}\left\{\mathbf{A}_{1 q}\right\}\end{array}\right]$

$\left.\left[\begin{array}{ccc}\operatorname{tr}\left\{\dot{\mathbf{A}}_{p 1}\right\} & \ddots & \vdots \\ \operatorname{tr}\left\{\dot{\mathbf{A}}_{p q}\right\}\end{array}\right]\right)$. Hence, $\operatorname{subtr}_{N}\{\cdot\}$ reduces the row and the column dimensionality by a factor $N$. Assuming that the different data streams are independent and possess the same autocorrelation function such that $\mathrm{E}\left\{\mathbf{x}^{(i)} \mathbf{x}^{(j) H}\right\}=\mathbf{R}_{x} \delta[i-j]$ for $i, j=1, \ldots, N_{t}$, the SNR at the MF equalizer output can then be written as

$$
\mathrm{SNR}_{o}=\frac{\mathbf{w}^{(a) H} \mathscr{H}\left(\mathbf{I}_{N_{t}} \otimes \mathbf{R}_{A}\right) \mathscr{H}^{H} \mathbf{w}^{(a)}}{\mathbf{w}^{(a) H} \mathbf{R}_{B} \mathbf{w}^{(a)}},
$$

where $\mathbf{R}_{A}=\operatorname{subtr}_{N}\left\{\mathbf{A R}_{x} \mathbf{A}^{H}\right\}$, and $\mathbf{R}_{B}=\operatorname{subtr}_{N}\left\{\left(\mathbf{I}_{N_{r}} \otimes\right.\right.$ $\left.\mathbf{B}) \mathbf{R}_{v}\left(\mathbf{I}_{N_{r}} \otimes \mathbf{B}^{H}\right)\right\}$, with $\mathbf{R}_{x}$ and $\mathbf{R}_{v}$ are the signal and noise autocorrelation matrices, respectively. For short we define $\mathbf{Q}=\mathbf{I}_{N_{t}} \otimes \mathbf{R}_{A}$.

Without loss of generality, the MF equalizer $\mathbf{w}^{(a)}$ can be forced to satisfy the constraint $\mathbf{w}^{(a) H} \mathbf{R}_{B} \mathbf{w}^{(a)}=1$, which can then obtained by solving the following constrained optimization problem

$$
\arg \max _{\mathbf{w}^{(a)}} \mathbf{w}^{(a) H} \mathscr{H} \mathbf{Q} \mathscr{H}^{H} \mathbf{w}^{(a)}, \quad \text { s.t. } \mathbf{w}^{(a) H} \mathbf{R}_{B} \mathbf{w}^{(a)}=1 .
$$

The problem in (21) is a generalized eigenvalue problem, with the MF equalizer coefficients are obtained as

$$
\mathbf{w}_{M F}^{(a)}=\operatorname{eig}_{\max }\left(\mathbf{R}_{B}^{-1} \mathscr{H} \mathbf{Q} \mathscr{H}^{H}\right)
$$

where $\operatorname{eig}_{\max }(\mathbf{A})$ is the eigenvector that corresponds to the maximum eigenvalue of the matrix A. For a white source $\left(\mathbf{R}_{x}=\sigma_{s}^{2} \mathbf{I}\right)$, and a white additive noise $\left(\mathbf{R}_{v}=\sigma_{n}^{2} \mathbf{I}\right)$, and a BEM resolution with $K=N$, the SNR at the output of the MF equalizer is obtained as

$$
\mathrm{SNR}_{o}=\frac{\mathbf{w}^{(a) H} \mathscr{H} \mathscr{H}^{H} \mathbf{w}^{(a)}}{\mathbf{w}^{(a) H} \mathbf{w}^{(a)}} \frac{\sigma_{s}^{2}}{\sigma_{n}^{2}}
$$

and hence the solution reduces now to

$$
\mathbf{w}_{M F}^{(a)}=\operatorname{eig}_{\max }\left\{\mathscr{H} \mathscr{H}^{H}\right\} .
$$

This is a special case of (22). However, for $K=2 N$, and provided that the source and the noise are white, the matrices $\mathbf{R}_{A}$, and $\mathbf{R}_{B}$ are not scalar multiple of the identity matrix. 
3.2. MMSE and ZF Equalizers. In the following, we will derive the linear MMSE and ZF equalizers. Starting with the linear MMSE equalizer, it can be determined by solving the following minimization problem:

$$
\arg \min _{\mathbf{w}^{(a)}} \mathrm{E}\left\{\left\|\hat{x}^{(a)}-\widetilde{\mathbf{Z}}_{d} \mathbf{x}^{(a)}\right\|^{2}\right\}
$$

where the multiplication with $\widetilde{\mathbf{Z}}_{d}$ accounts for the system decision delay. The minimization (25) can be equivalently written as

$$
\begin{aligned}
\arg \min _{\mathbf{w}^{(a)}} \mathbf{w}^{(a) H}\left(\mathscr{H} \mathbf{Q} \mathscr{H}^{H}+\mathbf{R}_{B}\right) \mathbf{w}^{(a)} \\
\quad-2 \mathfrak{R}\left\{\mathbf{w}^{(a) H} \mathscr{H}_{\mathbf{r}_{A}^{(a)}}^{(a)}+\operatorname{tr}\left\{\widetilde{\mathbf{Z}}_{d} \mathbf{R}_{x} \widetilde{\mathbf{Z}}_{d}^{T}\right\},\right.
\end{aligned}
$$

where $\mathbf{r}_{A}^{(a)}=\operatorname{subtr}_{N}\left\{\left(\mathbf{I}_{N_{t}} \otimes \mathbf{A}\right) \mathbf{R}_{x}\left(\mathbf{e}_{t}^{(a) T} \otimes \mathbf{I}_{N}\right) \widetilde{\mathbf{Z}}_{d}^{T}\right\}$, with $\mathbf{e}_{t}^{(a)}$ is an $N_{t}$ dimensional unity vector with one at the $a$ th position and zeros elsewhere. Solving for $\mathbf{w}^{(a)}$ in (26), we obtain

$$
\begin{aligned}
\mathbf{w}_{\mathrm{MMSE}}^{(a)} & =\left(\mathbf{R}_{B}+\mathscr{H} \mathbf{Q} \mathscr{H}^{H}\right)^{-1} \mathscr{H} \mathbf{r}_{A}^{(a)} \\
& =\mathbf{R}_{B}^{-1} \mathscr{H}\left(\mathscr{H}^{H} \mathbf{R}_{B}^{-1} \mathscr{H}+\mathbf{Q}^{-1}\right)^{-1} \mathbf{e}_{d}^{(a)},
\end{aligned}
$$

where $\mathbf{e}_{d}^{(a)}=\mathbf{e}_{t}^{(a)} \otimes \mathbf{e}_{d}$, with $\mathbf{e}_{d}$ is a $\left(Q+Q^{\prime}+1\right)(L+$ $\left.L^{\prime}+1\right)$ dimensional unity vector with one at position $(Q+$ $\left.Q^{\prime}\right)\left(L+L^{\prime}+1\right) / 2+d$. Note that $(28)$ is obtained from $(27)$ by applying the matrix inversion lemma $\left((\mathbf{A}+\mathbf{B C D})^{-1}=\right.$ $\left.\mathbf{A}^{-1}-\mathbf{A}^{-1} \mathbf{B}\left(\mathbf{D A}^{-1} \mathbf{B}+\mathbf{C}^{-1}\right)^{-1} \mathbf{D A}^{-1}\right)$, and using the fact that $\mathbf{Q}^{-1} \mathbf{r}_{A}^{(a)}=\mathbf{e}_{d}^{(a)}$.

The ZF solution can be obtained from the MMSE solution by setting the signal power to infinity. Hence, the ZF solution is obtained as

$$
\mathbf{w}_{Z F}^{(a)}=\mathbf{R}_{B}^{-1} \mathscr{H}\left(\mathscr{H}^{H} \mathbf{R}_{B}^{-1} \mathscr{H}\right)^{-1} \mathbf{e}_{d}^{(a)} .
$$

For the ZF solution to exist, the matrix $\mathscr{H}$ has to be of full column rank. A necessary condition for $\mathscr{H}$ to be of full column rank is that the inequality $N_{r}\left(Q^{\prime}+1\right)\left(L^{\prime}+1\right) \geq$ $N_{t}\left(Q+Q^{\prime}+1\right)\left(L+L^{\prime}+1\right)$ is satisfied. For sufficiently large $L^{\prime}$, and $Q^{\prime}$, this inequality is satisfied when the number of receive antennas is larger than the number of transmit antennas, that is, $N_{r} \geq N_{t}+1$. The MMSE equalizer always exists regardless of the number of receive antennas. However, the performance of the MMSE equalizer is largely improved if the above inequality is satisfied.

Obtaining the linear MMSE and ZF equalizers involves matrix inversion of size $P \times P$ with $P=N_{t}\left(Q+Q^{\prime}+1\right)(L+$ $\left.L^{\prime}+1\right)$. Therefore, the computational complexity of these equalizers requires $\mathcal{O}\left(P^{3}\right)$ Multiply-Add (MA) operations.

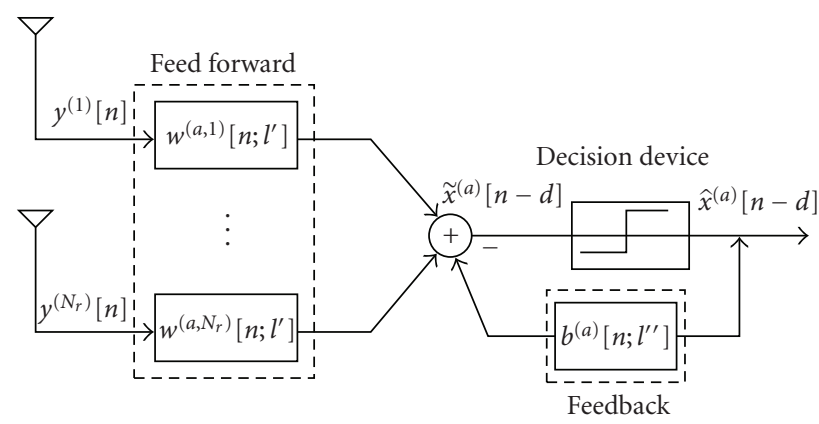

FIgURE 3: Time-varying FIR DFE-I for the ath antenna.

\section{Decision Feedback Equalization}

In this section we extend the results for linear equalization to decision feedback equalization. The DFE consists of two filters, a feed-forward filter and a feedback filter. The feedforward and feedback filters are again designed to be timevarying FIR filters. The time-varying FIR filters in the forward path are identical to the linear equalizers described in Section 3.2 (see Figure 2). The feedback filters have as their input the sequence of decisions on previously detected symbols. Given the extra degrees of freedom offered by the MIMO systems, we can devise two different scenarios. In one scenario, only previously estimated symbols from one transmit antenna are fed back to cancel/reduce ISI in the data stream of that particular transmit antenna. This scenario is referred to as DFE-I. In the other scenario, previously estimated symbols from all transmit antennas are fed back together to cancel/reduce ISI in the data stream of one particular transmit antenna. This scenario is referred to as DFE-II.

4.1. DFE-I. The DFE corresponding to this scenario is depicted in Figure 3. The feed-forward filters $w^{(a, r)}\left[n ; l^{\prime}\right]$ are identical to the linear equalizers developed in the previous section. For each data stream only one feedback filter is used to feedback previously detected symbols of that particular data stream. For the ath data stream, the feedback filter $b^{(a)}\left[n ; l^{\prime \prime}\right]$ is again designed as a time-varying FIR filter with order $L^{\prime \prime}$. Hence, an estimate of the transmitted symbol at time-index $n$ subject to the decision delay $d$ is obtained as

$$
\begin{aligned}
\tilde{x}^{(a)}[n-d]= & \sum_{r=1}^{N_{r}} \sum_{l^{\prime}=0}^{L^{\prime}} w^{(a, r) *}\left[n ; l^{\prime}\right] y^{(r)}\left[n-l^{\prime}\right] \\
& -\sum_{l^{\prime \prime}=\Delta}^{L^{\prime \prime}+\Delta} b^{(a) *}\left[n ; l^{\prime \prime}\right] \hat{x}^{(a)}\left[n-l^{\prime \prime}\right], \\
\hat{x}^{(a)}[n-d]= & Q\left(\tilde{x}^{(a)}[n-d]\right),
\end{aligned}
$$

where $\Delta \geq d+1$, and $Q(\cdot)$ is the quantizer used by the decision device. Based on the BEM (6), the time-varying FIR feedback filter on the $\left(l^{\prime \prime}\right)$ th tap can be written as

$$
b^{(a)}\left[n ; l^{\prime \prime}\right]=\sum_{q^{\prime \prime}=-Q^{\prime \prime} / 2}^{Q^{\prime \prime} / 2} b_{q^{\prime \prime}, l^{\prime \prime}}^{(a)} e^{-j 2 \pi n q^{\prime \prime} n / K},
$$


where $Q^{\prime \prime}$ is the number of time-varying basis functions. Substituting (31) into (30), and assuming past decisions are correct, yields the following:

$$
\begin{aligned}
\tilde{x}^{(a)}[n-d]= & \sum_{r=1}^{N_{r}} \sum_{q^{\prime}=-Q^{\prime} / 2}^{Q^{\prime} / 2} \sum_{l^{\prime}=0}^{L^{\prime}} w_{q^{\prime}, l^{\prime}}^{(a, r) *} e^{j 2 \pi q^{\prime} n / K} y^{(r)}\left[n-l^{\prime}\right] \\
& -\sum_{q^{\prime \prime}=-Q^{\prime \prime} / 2}^{Q^{\prime \prime} / 2} \sum_{l^{\prime \prime}=\Delta}^{L^{\prime \prime}+\Delta} b_{q^{\prime \prime}, l^{\prime}}^{(a) *} e^{j 2 \pi n q^{\prime \prime} n / K} x^{(a)}\left[n-l^{\prime \prime}\right] .
\end{aligned}
$$

With a block level formulation, (32) can be written as

$$
\begin{aligned}
\tilde{\mathbf{x}}^{(a)}= & \sum_{r=1}^{N_{r}} \sum_{q^{\prime}=-Q^{\prime} / 2}^{Q^{\prime} / 2} \sum_{l^{\prime}=0}^{L^{\prime}} w_{q^{\prime}, l^{\prime}}^{(a, r) *} \overline{\mathbf{D}}_{q^{\prime}} \overline{\mathbf{Z}}_{l^{\prime}} \mathbf{y}^{(r)} \\
& -\sum_{q^{\prime \prime}=-Q^{\prime \prime} / 2}^{Q^{\prime \prime} / 2} \sum_{l^{\prime \prime}=\Delta}^{L^{\prime \prime}+\Delta} b_{q^{\prime \prime}, l^{\prime \prime}}^{(a) *} \overline{\mathbf{D}}_{q^{\prime \prime}}, \tilde{\mathbf{Z}}_{l^{\prime \prime}} \mathbf{X}^{(a)} .
\end{aligned}
$$

The first part of (33) is similar to (12), and hence can be written as

$$
\begin{aligned}
\tilde{\mathbf{x}}^{(a)}= & \sum_{t=1}^{N_{t}} \sum_{p, k} f_{p, k}^{(a, t)} \overline{\mathbf{D}}_{p} \widetilde{\mathbf{Z}}_{k} \mathbf{\mathbf { x }}^{(t)}+\sum_{r=1}^{N_{r}} \sum_{q^{\prime}, l^{\prime}} w_{q^{\prime}, l^{\prime}}^{(a, r) *} \overline{\mathbf{D}}_{q^{\prime}} \overline{\mathbf{Z}}_{l^{\prime}} \mathbf{v}^{(r)} \\
& -\sum_{q^{\prime \prime}=-Q^{\prime \prime} / 2}^{Q^{\prime \prime} / 2} \sum_{l^{\prime \prime}=\Delta}^{L^{\prime \prime}+\Delta} b_{q^{\prime \prime}, l^{\prime \prime}}^{(a) *} \overline{\mathbf{D}}_{q^{\prime \prime}} \widetilde{\mathbf{Z}}_{l^{\prime}} \mathbf{X}^{(a)} .
\end{aligned}
$$

Assuming the feedback filter is designed such that $Q^{\prime \prime} \leq Q+$ $Q^{\prime}$, and $L^{\prime \prime}+\Delta \leq L+L^{\prime}$, then (34) can be written as

$$
\begin{aligned}
\tilde{\mathbf{x}}^{(a)}= & \sum_{t=1}^{N_{t}} \sum_{p, k} f_{p, k}^{(a, t)} \overline{\mathbf{D}}_{p} \widetilde{\mathbf{Z}}_{k} \mathbf{x}^{(t)}+\sum_{r=1}^{N_{r}} \sum_{q^{\prime}, l^{\prime}} w_{q^{\prime}, l^{\prime}}^{(a, r) *} \overline{\mathbf{D}}_{q^{\prime}} \overline{\mathbf{Z}}_{l^{\prime}} \mathbf{v}^{(r)} \\
& -\sum_{p, k} \tilde{b}_{p, k}^{(a) *} \overline{\mathbf{D}}_{p} \widetilde{\mathbf{Z}}_{k} \mathbf{x}^{(a)}
\end{aligned}
$$

where $\tilde{b}_{p, k}^{(a)}=b_{q^{\prime \prime}, l^{\prime \prime}}^{(a)} \delta\left[p-q^{\prime \prime}\right] \delta\left[k-l^{\prime \prime}\right]$, that is, zeros are appended in the feedback filter BEM coefficients such that the span of the BEM coefficients of the feedback filter matches that of the sequence $f_{p, k}^{(a)}$. Hence, (35) can finally be written in a compact vector-matrix form as

$$
\begin{aligned}
\tilde{\mathbf{x}}^{(a)}= & \left(\mathbf{f}^{(a) T} \otimes \mathbf{I}_{N}\right)\left(\mathbf{I}_{N_{t}} \otimes \mathbf{A}\right) \mathbf{x} \\
& +\left(\mathbf{w}^{(a) H} \otimes \mathbf{I}_{N}\right)\left(\mathbf{I}_{N_{r}} \otimes \mathbf{B}\right)-\left(\widetilde{\mathbf{b}}^{(a) H} \otimes \mathbf{I}_{N}\right) \mathbf{A} \mathbf{x}^{(a)} .
\end{aligned}
$$

Defining $\mathbf{b}^{(a)}=\left[b_{-Q^{\prime \prime} / 2,0}^{(a)}, \ldots, b_{-Q^{\prime \prime} / 2, L^{\prime \prime}}^{(a)}, \ldots, b_{Q^{\prime \prime} / 2, L^{\prime \prime}}^{(a)}\right]^{T}$, then $\widetilde{\mathbf{b}}^{(a)}$ is obtained as $\widetilde{\mathbf{b}}^{(a)}=\mathbf{P} \mathbf{b}^{(a)}$, where $\mathbf{P}$ is a $\left(Q+Q^{\prime}+1\right)(L+$ $\left.L^{\prime}+1\right) \times\left(Q^{\prime \prime}+1\right)\left(L^{\prime \prime}+1\right)$ selection matrix defined as

$$
\mathbf{P}=\left[\begin{array}{c}
\mathbf{0}_{\left(Q+Q^{\prime}-Q^{\prime \prime}\right) / 2\left(L+L^{\prime}+1\right) \times\left(Q^{\prime \prime}+1\right)\left(L^{\prime \prime}+1\right)} \\
\mathbf{I}_{\left(Q^{\prime \prime}+1\right)} \otimes \mathbf{J} \\
\mathbf{0}_{\left(Q+Q^{\prime}-Q^{\prime \prime}\right) / 2\left(L+L^{\prime}+1\right) \times\left(Q^{\prime \prime}+1\right)\left(L^{\prime \prime}+1\right)}
\end{array}\right],
$$

with the $\left(L+L^{\prime}+1\right) \times\left(L^{\prime \prime}+1\right)$ matrix $\mathbf{J}$ defined as

$$
\mathbf{J}=\left[\begin{array}{c}
\mathbf{0}_{\Delta \times\left(L^{\prime \prime}+1\right)} \\
\mathbf{I}_{L^{\prime \prime}+1} \\
\mathbf{0}_{\left(L+L^{\prime}-L^{\prime \prime}-\Delta\right) \times\left(L^{\prime \prime}+1\right)}
\end{array}\right],
$$

where $\Delta$ is restricted to $\Delta=d+1$ to simplify the forthcoming analysis. Assuming past decisions are correct, the MMSEDFE is then obtained by minimizing the error across the decision device as

$$
\arg \min _{\mathbf{w}^{(a)}, \mathbf{b}^{(a)}}\left\|\tilde{\mathbf{x}}^{(a)}-\tilde{\mathbf{Z}}_{d} \mathbf{X}^{(a)}\right\|^{2} .
$$

Using (34), the mean square-error (MSE) function MSE = $\left\|\mathbf{x}^{(a)}-\widetilde{\mathbf{Z}}_{d} \mathbf{x}^{(a)}\right\|^{2}$ can be written as

$$
\begin{aligned}
\mathrm{MSE}= & \mathbf{w}^{(a) H}\left(\mathscr{H} \mathbf{Q} \mathscr{H}^{H}+\mathbf{R}_{B}\right) \mathbf{w}^{(a)} \\
& -2 \Re\left\{\mathbf{w}^{(a) H} \mathscr{H}\left(\mathbf{e}_{a} \otimes \mathbf{R}_{A}\right)\left(\tilde{\mathbf{b}}^{(a)}+\mathbf{r}_{A}^{(a)}\right)\right\} \\
& +\left(\widetilde{\mathbf{b}}^{(a)}+\mathbf{e}_{d}\right)^{H} \mathbf{R}_{A}\left(\widetilde{\mathbf{b}}^{(a)}+\mathbf{e}_{d}\right) .
\end{aligned}
$$

Solving for the feed-forward coefficients $\mathbf{w}^{(a)}$ in (40) such that $\partial \mathrm{MSE} / \partial \mathbf{w}^{(a)}=\mathbf{0}$, we obtain

$$
\mathbf{w}^{(a)}=\mathbf{R}_{B}^{-1} \mathscr{H}\left(\mathbf{Q}^{-1}+\mathscr{H}^{H} \mathbf{R}_{B}^{-1} \mathscr{H}\right)^{-1} \mathbf{I}_{\Delta}^{(a)}\left(\tilde{\mathbf{b}}^{(a)}+\mathbf{e}_{d}\right),
$$

where $\mathbf{I}_{\Delta}^{(a)}=\mathbf{e}_{t}^{(a)} \otimes \mathbf{I}_{\left(Q+Q^{\prime}+1\right)\left(L+L^{\prime}+1\right)}$. Note that, for no feedback the feed-forward filter in (41) reduces to the MMSE filter solution (28). Substituting (41) into (40), yields the following MSE:

$$
\operatorname{MSE}=\left(\widetilde{\mathbf{b}}^{(a)}+\mathbf{e}_{d}\right)^{H} \mathbf{R}_{\perp}^{(a)}\left(\widetilde{\mathbf{b}}^{(a)}+\mathbf{e}_{d}\right),
$$

where $\mathbf{R}_{\perp}^{(a)}$ is given as

$$
\mathbf{R}_{\perp}^{(a)}=\mathbf{R}_{A}-\mathbf{I}_{\Delta}^{(a) T}\left(\mathbf{Q}^{-1}+\mathscr{H}^{H} \mathbf{R}_{B}^{-1} \mathscr{H}\right)^{-1} \mathscr{H}^{H} \mathbf{R}_{B}^{-1} \mathscr{H} \mathbf{Q} \mathbf{I}_{\Delta}^{(a)} .
$$

The feedback BEM coefficients are then obtained by solving the following equation:

$$
\arg \min _{\mathbf{b}^{(a)}} \text { MSE. }
$$

To solve for the feedback filter BEM coefficients, we define $\mathbf{u}^{(a)}=\left[\mathbf{b}_{-Q^{\prime \prime} / 2}^{(a) T}, \ldots, \mathbf{b}_{-1}^{(a) T}, 1, \mathbf{b}_{0}^{(a) T}, \ldots, \mathbf{b}_{Q^{\prime \prime} / 2}^{(a) T}\right]^{T}$, with $\mathbf{b}_{q^{\prime \prime}}^{(a)}=$ $\left[b_{q^{\prime \prime}, 0}^{(a)}, \ldots, b_{q^{\prime \prime}, L^{\prime \prime}}^{(a)}\right]^{T}$. Hence, (44) is equivalent to the following constrained quadratic minimization problem

$$
\arg \min _{\mathbf{u}^{(a)}} \mathbf{u}^{(a) H} \mathbf{R}_{\Delta}^{(a)} \mathbf{u}^{(a)}, \quad \text { s.t. } \mathbf{u}^{(a) H} \mathbf{e}_{0}=1,
$$




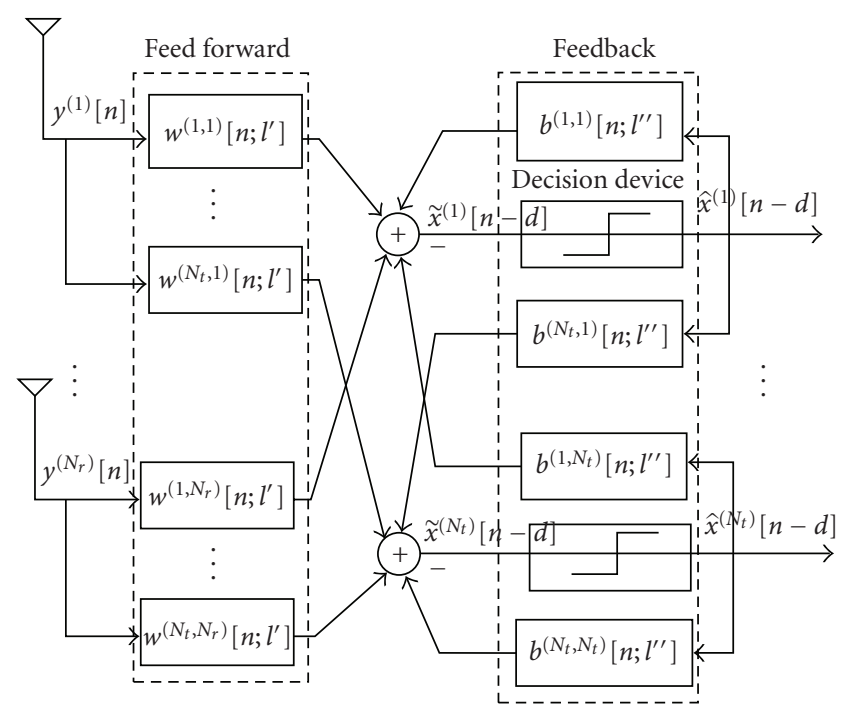

FIgURE 4: Time-varying FIR DFE-II for MIMO transmission.

where $\mathbf{R}_{\Delta}^{(a)}=\widetilde{\mathbf{P}}^{T} \mathbf{R}_{\perp}^{(a)} \widetilde{\mathbf{P}}$ where $\widetilde{\mathbf{P}}$ is a $\left(Q+Q^{\prime}+1\right)\left(L+L^{\prime}+1\right) \times$ $\left(\left(Q^{\prime \prime}+1\right)\left(L^{\prime \prime}+1\right)+1\right)$ selection matrix defined as

$$
\widetilde{\mathbf{P}}=\left[\begin{array}{c}
\mathbf{0}_{\left(Q+Q^{\prime}-Q^{\prime \prime}\right) / 2\left(L+L^{\prime}+1\right) \times\left(Q^{\prime \prime}+1\right)\left(L^{\prime \prime}+1\right)+1} \\
\left(\mathbf{I}_{Q^{\prime \prime} / 2} \otimes \mathbf{J}\right) \oplus \widetilde{\mathbf{J}} \oplus\left(\mathbf{I}_{Q^{\prime \prime} / 2} \otimes \mathbf{J}\right) \\
\mathbf{0}_{\left(Q+Q^{\prime}-Q^{\prime \prime}\right) / 2\left(L+L^{\prime}+1\right) \times\left(Q^{\prime \prime}+1\right)\left(L^{\prime \prime}+1\right)+1}
\end{array}\right],
$$

with $\tilde{\mathbf{J}}$ is defined as

$$
\tilde{\mathbf{J}}=\left[\begin{array}{c}
\mathbf{0}_{d \times\left(L^{\prime \prime}+2\right)} \\
\mathbf{I}_{L^{\prime \prime}+2} \\
\mathbf{0}_{\left(L+L^{\prime}-L^{\prime \prime}-\Delta\right) \times\left(L^{\prime \prime}+2\right)}
\end{array}\right],
$$

and $\mathbf{e}_{0}$ is a $\left(Q^{\prime \prime}+1\right)\left(L^{\prime \prime}+1\right)+1$ dimensional unity vector with one in position $Q^{\prime \prime}\left(L^{\prime \prime}+1\right) / 2+1$. The feedback filter coefficients are then obtained by solving the constrained quadratic minimization problem (45) as

$$
\mathbf{u}^{(a)}=\frac{\mathbf{R}_{\Delta}^{(a)-1} \mathbf{e}_{0}}{\mathbf{e}_{0}^{T} \mathbf{R}_{\Delta}^{(a)-T} \mathbf{e}_{0}}
$$

The computational complexity of the feed-forward filter coefficients requires matrix inversion of size $P=N_{t}(Q+$ $\left.Q^{\prime}+1\right)\left(L+L^{\prime}+1\right)$, and hence the complexity is $\mathcal{O}\left(P^{3}\right)$. Computing the feedback filter coefficients requires matrix inversion of size $\left(Q^{\prime \prime}+1\right)\left(L^{\prime \prime}+1\right)+1$, which requires complexity $\mathcal{O}\left(\left(\left(Q^{\prime \prime}+1\right)\left(L^{\prime \prime}+1\right)+1\right)^{3}\right)$. In most of the cases, the feedback filter order and number of time-varying basis functions are smaller than the feed-forward filter order and number of basis functions, that is, $\left(Q^{\prime \prime}+1\right)\left(L^{\prime \prime}+1\right) \ll$ $\left(Q+Q^{\prime}+1\right)\left(L+L^{\prime}+1\right)$. Hence, the computational complexity of the DFE of this scenario is dominated by the computation of the feed-forward filter $\mathcal{O}\left(P^{3}\right)$, which is exactly the same computational complexity of the linear MMSE or ZF filters. However, the overall complexity is slightly larger for this DFE than the linear equalizers.
4.2. DFE-II. The DFE corresponding to this scenario is depicted in Figure 4. In this scenario, past estimates of symbols on all data streams are made available to cancel/reduce ISI. Thus, for each data stream $N_{t}$ feedback equalizers are employed. Denote the feedback filter that feedback past decisions of the $t$ th antenna data stream to cancel ISI on the ath antenna data stream as $b^{(a, t)}\left[n ; l^{\prime \prime}\right]$. Assuming past decisions are correct, an estimate of the transmitted symbols at time-index $n$ subject to the decision delay $d$ can be obtained as

$$
\begin{aligned}
\tilde{x}^{(a)}[n-d]= & \sum_{r=1}^{N_{r}} \sum_{q^{\prime}=-Q^{\prime} / 2}^{Q^{\prime} / 2} \sum_{l^{\prime}=0}^{L^{\prime}} w_{q^{\prime}, l^{\prime}}^{(a, r) *} e^{j 2 \pi q^{\prime} n / K} y^{(r)}\left[n-l^{\prime}\right] \\
& -\sum_{t=1}^{N_{t}} \sum_{q^{\prime \prime}=-Q^{\prime \prime} / 2}^{Q^{\prime \prime} / 2} \sum_{l^{\prime \prime}=\Delta}^{L^{\prime \prime}+\Delta} b_{q^{\prime \prime}, l^{\prime \prime}}^{(a, t) *} e^{j 2 \pi n q^{\prime \prime} n / K} x^{(t)}\left[n-l^{\prime \prime}\right] .
\end{aligned}
$$

With a block level formulation, (49) can be written as

$$
\begin{aligned}
\tilde{\mathbf{x}}^{(a)}= & \sum_{t=1}^{N_{t}} \sum_{p, k} f_{p, k}^{(a, t)} \overline{\mathbf{D}}_{p} \widetilde{\mathbf{Z}}_{k} \mathbf{x}^{(t)}+\sum_{r=1}^{N_{r}} \sum_{q^{\prime}, l^{\prime}} w_{q^{\prime}, l^{\prime}}^{(a, r) *} \overline{\mathbf{D}}_{q^{\prime}} \overline{\mathbf{Z}}_{l^{\prime}} \mathbf{v}^{(r)} \\
& -\sum_{t=1}^{N_{t}} \sum_{q^{\prime \prime}=-Q^{\prime \prime} / 2}^{Q^{\prime \prime} / 2} \sum_{l^{\prime \prime}=\Delta}^{L^{\prime \prime}+\Delta} b_{q^{\prime \prime}, l^{\prime \prime}}^{(a, t) *} \overline{\mathbf{D}}_{q^{\prime \prime}} \widetilde{\mathbf{Z}}_{l^{\prime \prime}} \mathbf{x}^{(t)}
\end{aligned}
$$

Assuming the feedback filters are designed such that $Q^{\prime \prime} \leq$ $Q+Q^{\prime}$ and $L^{\prime \prime}+\Delta \leq L+L^{\prime}$, then we can write (50) as

$$
\begin{aligned}
\tilde{\mathbf{x}}^{(a)}= & \sum_{t=1}^{N_{t}} \sum_{p, k} f_{p, k}^{(a, t)} \overline{\mathbf{D}}_{p} \widetilde{\mathbf{Z}}_{k} \mathbf{x}^{(t)}+\sum_{r=1}^{N_{r}} \sum_{q^{\prime}, l^{\prime}} w_{q^{\prime}, l^{\prime}}^{(a, r) *} \overline{\mathbf{D}}_{q^{\prime}} \overline{\mathbf{Z}}_{l^{\prime}} \mathbf{v}^{(r)} \\
& -\sum_{t=1}^{N_{t}} \sum_{p, k} \tilde{b}_{p, k}^{(a, t) *} \overline{\mathbf{D}}_{p} \tilde{\mathbf{Z}}_{k} \mathbf{x}^{(t)}
\end{aligned}
$$

where $\tilde{b}_{p, k}^{(a, t)}=b_{q^{\prime \prime}, l^{\prime \prime}}^{(a, t)} \delta\left[p-q^{\prime \prime}\right] \delta\left[k-l^{\prime \prime}\right]$. In a compact vector form, (51) can finally be written as

$$
\begin{aligned}
\tilde{\mathbf{x}}^{(a)}= & \left(\mathbf{f}^{(a) T} \otimes \mathbf{I}_{N}\right)\left(\mathbf{I}_{N_{t}} \otimes \mathbf{A}\right) \mathbf{x}+\left(\mathbf{w}^{(a) H} \otimes \mathbf{I}_{N}\right)\left(\mathbf{I}_{N_{r}} \otimes \mathbf{B}\right) \\
& -\left(\tilde{\mathbf{b}}^{(a) H} \otimes \mathbf{I}_{N}\right)\left(\mathbf{I}_{N_{t}} \otimes \mathbf{A}\right) \mathbf{x},
\end{aligned}
$$

where $\tilde{\mathbf{b}}^{(a)}=\left[\tilde{\mathbf{b}}^{(a, 1) T}, \ldots, \tilde{\mathbf{b}}^{\left(a, N_{t}\right) T}\right]^{T}$, with $\tilde{\mathbf{b}}^{(a, t)}=\mathbf{P b}^{(a, t)}$, and $\mathbf{b}^{(a, t)}=\left[b_{-Q^{\prime \prime} / 2,0}^{(a, t)}, \ldots, b_{-Q^{\prime \prime} / 2, L^{\prime \prime}}^{(a, t)}, \ldots, b_{Q^{\prime \prime} / 2, L^{\prime \prime}}^{(a, t)}\right]^{T}$. The MSE across the decision device can then be obtained as

$$
\begin{aligned}
\mathrm{MSE}= & \left\|\tilde{\mathbf{x}}^{(a)}-\widetilde{\mathbf{Z}}_{d} \mathbf{x}^{(a)}\right\|^{2} \\
= & \mathbf{w}^{(a) H}\left(\mathscr{H} \mathbf{Q} \mathscr{H}^{H}+\mathbf{R}_{B}\right) \mathbf{w}^{(a)} \\
& -2 \mathfrak{R}\left\{\mathbf{w}^{(a) H} \boldsymbol{H} \mathbf{Q}\left(\tilde{\mathbf{b}}^{(a)}+\mathbf{e}_{d}^{(a)}\right)\right\} \\
& +\left(\tilde{\mathbf{b}}^{(a)}+\mathbf{e}_{d}^{(a)}\right)^{H} \mathbf{Q}\left(\tilde{\mathbf{b}}^{(a)}+\mathbf{e}_{d}^{(a)}\right) .
\end{aligned}
$$


Solving for the feed-forward coefficients in (53) such that $\partial \mathrm{MSE} / \partial \mathbf{w}^{(a)}=\mathbf{0}$, we obtain

$$
\mathbf{w}^{(a)}=\mathbf{R}_{B}^{-1} \mathscr{H}\left(\mathbf{Q}^{-1}+\mathscr{H}^{H} \mathbf{R}_{B}^{-1} \mathscr{H}\right)^{-1}\left(\widetilde{\mathbf{b}}^{(a)}+\mathbf{e}_{d}^{(a)}\right) .
$$

Substituting for the feed-forward coefficients (54) into (53) yields the following MSE

$$
\operatorname{MSE}=\left(\widetilde{\mathbf{b}}^{(a)}+\mathbf{e}_{d}^{(a)}\right)^{H} \mathbf{R}_{\perp}\left(\widetilde{\mathbf{b}}^{(a)}+\mathbf{e}_{d}^{(a)}\right)
$$

where the matrix $\mathbf{R}_{\perp}$ is given as

$$
\begin{aligned}
\mathbf{R}_{\perp} & =\mathbf{Q}-\mathbf{Q} \mathscr{H}^{H}\left(\mathscr{H} \mathbf{Q} \mathscr{H}^{H}+\mathbf{R}_{B}\right)^{-1} \mathscr{H}^{H} \mathbf{Q} \\
& =\left(\mathscr{H}^{H} \mathbf{R}_{B}^{-1} \mathscr{H}+\mathbf{Q}^{-1}\right)^{-1} .
\end{aligned}
$$

Defining $\mathbf{u}^{(a)}=\left[\mathbf{u}^{(a, 1) T}, \ldots, \mathbf{u}^{\left(a, N_{t}\right) T}\right]^{T}$, where

$$
\mathbf{u}^{(a, t)}= \begin{cases}\mathbf{b}^{(a, t)} & t \neq a \\ {\left[\mathbf{b}_{-Q^{\prime \prime} / 2}^{(a, t) T}, \ldots, 1, \mathbf{b}_{0}^{(a, t) T}, \ldots, \mathbf{b}_{Q^{\prime \prime} / 2}^{(a, t) T}\right]^{T}} & t=a,\end{cases}
$$

the MMSE-DFE feedback filter BEM coefficients are obtained by solving for the following constrained quadratic minimization problem:

$$
\arg \min _{\mathbf{u}^{(a)}} \mathbf{u}^{(a) H} \widetilde{\mathbf{R}}_{\Delta}^{(a)} \mathbf{u}^{(a)}, \quad \text { s.t. } \mathbf{u}^{(a) H} \mathbf{e}_{0}^{(a)}=1,
$$

where $\widetilde{\mathbf{R}}_{\Delta}^{(a)}=\mathbf{P}^{(a) T} \mathbf{R}_{\perp} \mathbf{P}^{(a)}$, with

$$
\mathbf{P}^{(a)}=\mathbf{P} \oplus \ldots \underbrace{\widetilde{\mathbf{P}}}_{\text {ath position }} \oplus \ldots \mathbf{P}
$$

and $\mathbf{e}_{0}^{(a)}$ is an $N_{t}\left(Q^{\prime \prime}+1\right)\left(L^{\prime \prime}+1\right)+1$ dimensional unity vector with one in position $(a-1)\left(Q^{\prime \prime}+1\right)\left(L^{\prime \prime}+1\right)+Q^{\prime \prime} / 2\left(L^{\prime \prime}+1\right)+1$. The feedback filter coefficients are finally obtained as

$$
\mathbf{u}^{(a)}=\frac{\widetilde{\mathbf{R}}_{\Delta}^{(a)-1} \mathbf{e}_{0}^{(a)}}{\mathbf{e}_{0}^{(a) T} \widetilde{\mathbf{R}}_{\Delta}^{(a)-T} \mathbf{e}_{0}^{(a)}}
$$

The computational complexity of the feed-forward filter coefficients of this scenario requires matrix inversion of size $P \times P$, which requires $\mathcal{O}\left(P^{3}\right)$ MA operations. This complexity is the same complexity associated with computing the feedforward filter coefficients of DFE-I scenario and the linear equalizer. Computing the feedback filter coefficients requires matrix inversion of size $N_{t}\left(Q^{\prime \prime}+1\right)\left(L^{\prime \prime}+1\right)+1 \times N_{t}\left(Q^{\prime \prime}+\right.$ $1)\left(L^{\prime \prime}+1\right)+1$, which requires $\mathcal{O}\left(\left(N_{t}\left(Q^{\prime \prime}+1\right)\left(L^{\prime \prime}+1\right)+1\right)^{3}\right) \mathrm{MA}$ operations. In this sense, the computational complexity of DFE-II for the feedback part is $N_{t}^{3}$ times the computational complexity of DFE-I. Provided that $\left(Q^{\prime \prime}+1\right)\left(L^{\prime \prime}+1\right) \ll$ $\left(Q+Q^{\prime}+1\right)\left(L+L^{\prime}+1\right)$, the computational complexity is still dominated by the computation of the feed-forward filter. However, the overall computational complexity for DFE-II is the highest among all devised equalizers.

\section{Simulation Results}

In this section we present some simulation results for the proposed equalization techniques for MIMO transmission over doubly selective channels. In the simulations, uncoded Quadrature Phase Shift Keying (QPSK) modulation is used. The channel is assumed to be perfectly known at the receiver. The BEM coefficients are then obtained by least-squares (LS) fitting the true underlying channel with the BEM. The performance of the proposed equalization techniques under channel estimation errors is outside the scope of this paper, and a topic of further investigation. The channel taps are simulated as i.i.d random variables, correlated in time according to Jakes' model with correlation function $r_{h}[n]=$ $J_{0}\left(2 \pi n f_{\max } T\right)$, where $J_{0}$ is the zeroth-order Bessel function of the first kind, $f_{\max } T$ is the maximum normalized Doppler spread. Two channel setups are used. One with order $L=3$, and $f_{\max } T=0.001$, and another setup with order $L=6$, and normalized maximum Doppler spread $f_{\max } T=5 \times 10^{-4}$. A BEM window size $N=800$ is considered all over the simulations. The BEM resolution is chosen such that $K=N$ and $K=2 N$. We measure the performance in terms of bit error rate (BER) versus signal-to-noise ratio (SNR). The SNR is defined as $(L+1) E_{s} / \sigma_{n}^{2}$, where $E_{s}$ is the transmitted symbol energy, and $\sigma_{n}^{2}$ is the additive white Gaussian noise variance. In all the simulations the decision delay is taken as $d=$ $\left(L+L^{\prime}\right) / 2+1$, and the approximation $\mathbf{R}_{A} \approx \sigma_{x}^{2} \mathbf{I}_{\left(Q+Q^{\prime}+1\right)\left(L+L^{\prime}+1\right)}$, and $\mathbf{R}_{B} \approx \sigma_{n}^{2} \mathbf{I}_{\left(Q^{\prime}+1\right)\left(L^{\prime}+1\right)}$ are used. It is worth mentioning here that the BEM approximated channel is used for the equalizers design, but the true channel is used for BER simulations, which will be subject to channel modeling error.

5.1. Channel Setup-I. In this setup the number of basis functions is $Q=2$ for $K=N$, and $Q=4$ for $K=2 N$. Two MIMO setups are considered; a first setup with $N_{t} \times N_{r}=$ $2 \times 4$, and a second setup with $N_{t} \times N_{r}=2 \times 2$. In the first setup, a linear ZF equalizer may exist, and therefore it can be evaluated against the linear MMSE equalizer and DFE. In the second setup, only the linear MMSE equalizer and the DFE are evaluated.

There are many parameters to tune in the simulations, and to choose the optimal parameter set is a difficult task. In this sense we divided the problem into two sets. In the first set we fixed $L^{\prime \prime}$ and $Q^{\prime \prime}$ and vary $L^{\prime}$ and $Q^{\prime}$ at a fixed SNR value. In the second we obtained the optimal (best) $L^{\prime}$ and $Q^{\prime}$ and varied the feedback parameters $L^{\prime \prime}$ and $Q^{\prime \prime}$. In this sense, the BER performance is evaluated as a function of the feed-forward filter order $L^{\prime}$ and number of timevarying basis functions $Q^{\prime}$ at a fixed $S N R=8 \mathrm{~dB}$. The the feedback filters parameters are set at $Q^{\prime \prime}=4$, and $L^{\prime \prime}=3$, which are the same as the channel BEM parameters. The simulation results corresponding to this setup are shown in Figure 5. For $K=N$ the BER is significantly improved for $L^{\prime} \geq 4$ which corresponds to the case where ZF solution exists. For $K=2 N$, the BER improves by increasing $L^{\prime}$, for a value of $L^{\prime} \geq 8$, the improvement in the BER becomes marginal. For this reason the order of the feed-forward filters is chosen to be $L^{\prime}=8$. Similarly the BER performance improves by increasing $Q^{\prime}$. Clearly there is no significant 


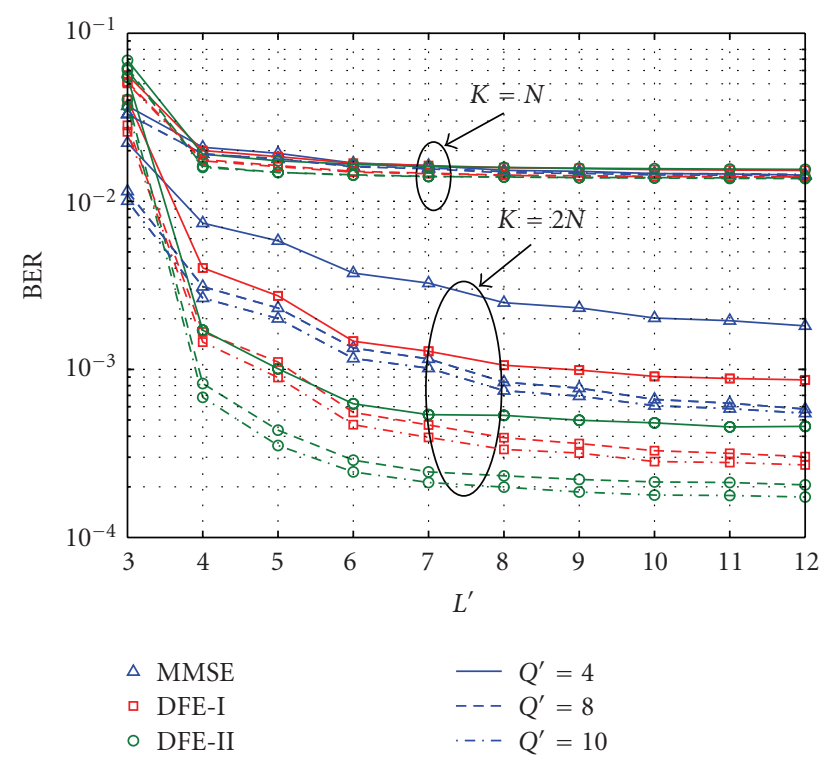

FIgURe 5: BER versus $Q^{\prime}$ and $L^{\prime}$ for $N_{t} \times N_{r}=2 \times 4$, SNR $=8 \mathrm{~dB}$.

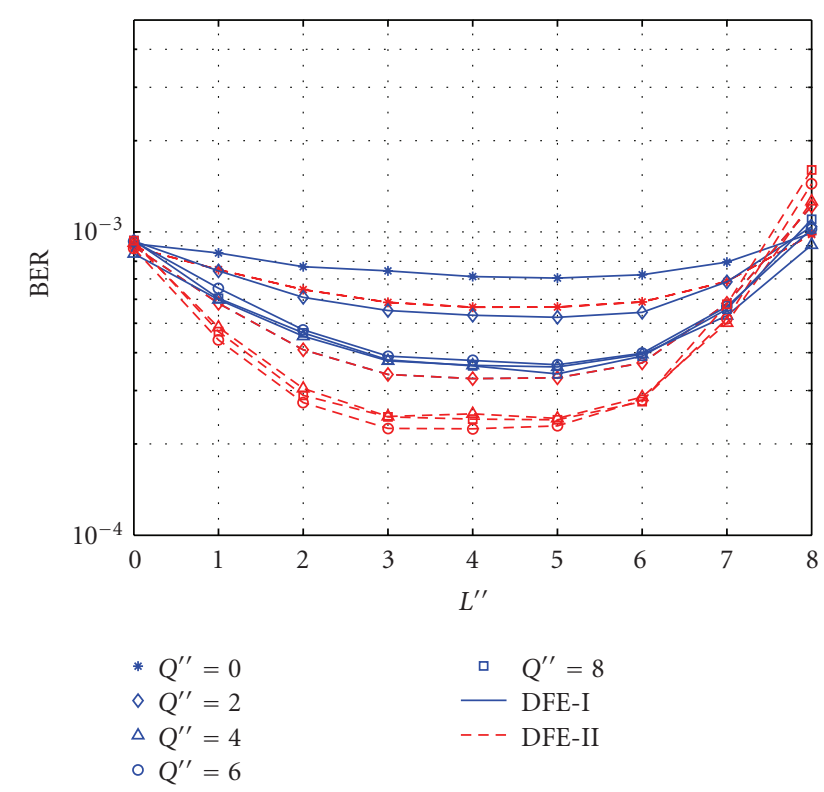

FIgURe 6: BER versus $Q^{\prime \prime}$ and $L^{\prime \prime}$ for $N_{t} \times N_{r}=2 \times 4, \mathrm{SNR}=8 \mathrm{~dB}$.

difference in BER performance for $Q^{\prime}=8$ compared to $Q^{\prime}=10$. Taking complexity into account, $Q^{\prime}$ is set at $Q^{\prime}=8$. Setting $L^{\prime}=8$, and $Q^{\prime}=8$, we also vary the feedback filter parameters $Q^{\prime \prime}$, and $L^{\prime \prime}$ for the DFE-I and DFE-II scenarios. At an SNR $=8 \mathrm{~dB}$, the simulation results are shown in Figure 6. The BER performance improves by increasing $Q^{\prime \prime}$. For DFE-I scenario the BER performance significantly improves by increasing $Q^{\prime \prime}$ up to $Q^{\prime \prime}=4$, for which the performance is rather steady. For DFE-II scenario, similar results are observed, except that the performance is slightly best for $Q^{\prime \prime}=6$. With regard to feedback filters order $L^{\prime \prime}$, the BER performance is significantly improved for $3 \leq L^{\prime \prime} \leq 5$. Therefore, the feedback filter order is set at

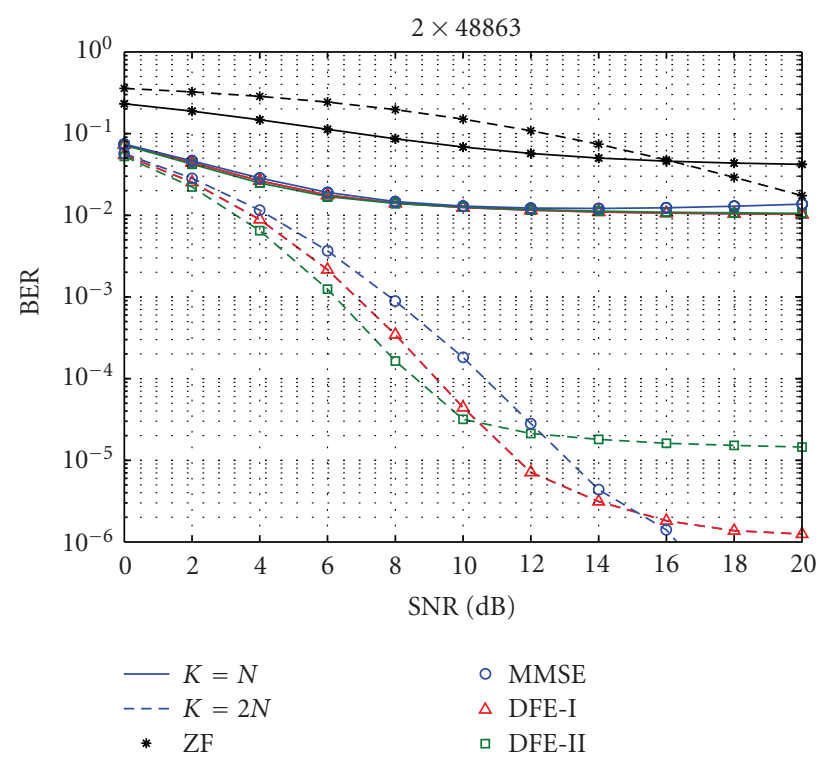

FIgURE 7: BER versus SNR for $N_{t} \times N_{r}=2 \times 4$.

$L^{\prime \prime}=3$, and the number of time-varying basis functions is set at $Q^{\prime \prime}=6$. These specifications of the feed-forward filters are sufficient for the linear ZF equalizer to exist. The simulation results for the BER versus SNR corresponding to this MIMO setup are shown in Figure 7. For $K=N$, the equalizers suffer from an early error floor at BER $=10^{-2}$ except for the linear ZF equalizer which shows a higher error floor at BER $=4 \times 10^{-2}$. For $K=2 N$, the BER curves for DFE-I and DFE-II show an error floor at BER $=1.3 \times$ $10^{-6}$ and BER $=1.5 \times 10^{-5}$, respectively. Benchmarking at $\mathrm{BER}=10^{-4}$, DFE-I, and DFE-II outperform the linear MMSE and ZF equalizers. An SNR gain of $1.3 \mathrm{~dB}$ and $2 \mathrm{~dB}$ is observed for DFE-I and DFE-II over the linear MMSE equalizer, respectively. The performance of the linear $\mathrm{ZF}$ equalizer is very marginal. The performance of DFE-II suffers from a higher error floor than DFE-I, this actually due to the fact that DFE-II is more prone to error propagation than DFE-I, which is clear from the BER figures at high SNR values, where error propagation would become the limiting factor rather than the noise power. This can also be explained based on the structure of each equalizer. Based on their structure, DFE-I feeds back past decisions on only one data stream to cancel/reduce ISI on that particular data stream, while DFE-II feeds back past decisions on all data streams. As such, for DFE-I, incorrect past decisions on one data stream will influence subsequent decisions on only that data stream, while incorrect decisions on one or more data streams for DFE-II will influence subsequent decisions on the other data streams, and hence more likely to propagate. Therefore, DFE-II based on its structure is more vulnerable to error propagation than DFE-I.

For the second setup, the simulation results are shown in Figure 8 . The feed-forward filters are designed to have order $L^{\prime}=12$, and the number of time-varying basis functions $Q^{\prime}=12$. For the decision feedback equalizers the feedback filters are designed to have order $L^{\prime \prime}=6$, and number of 


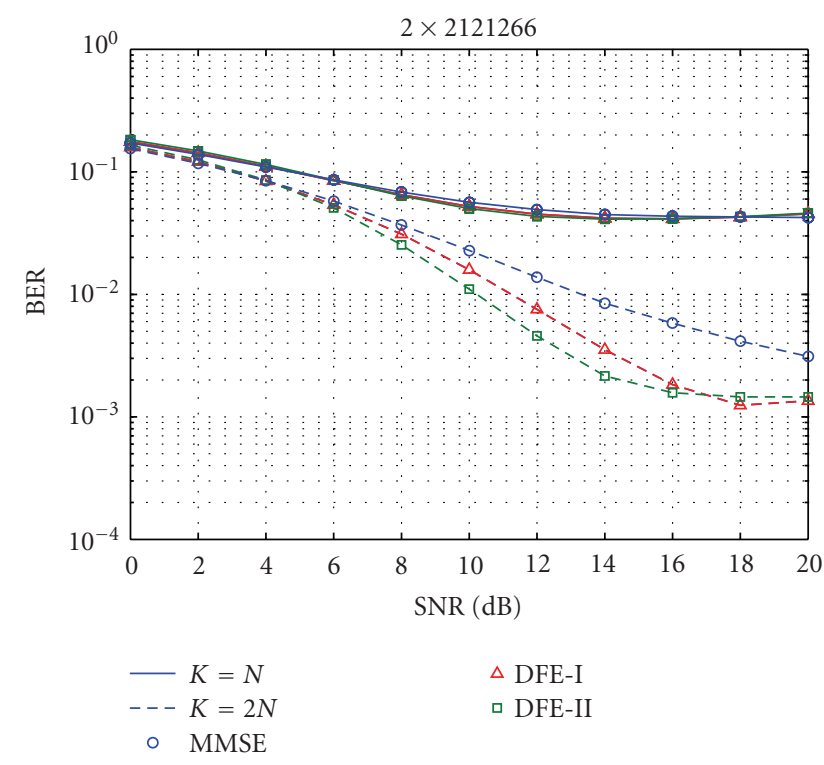

FIgURE 8: BER versus SNR for $N_{t} \times N_{r}=2 \times 2$.

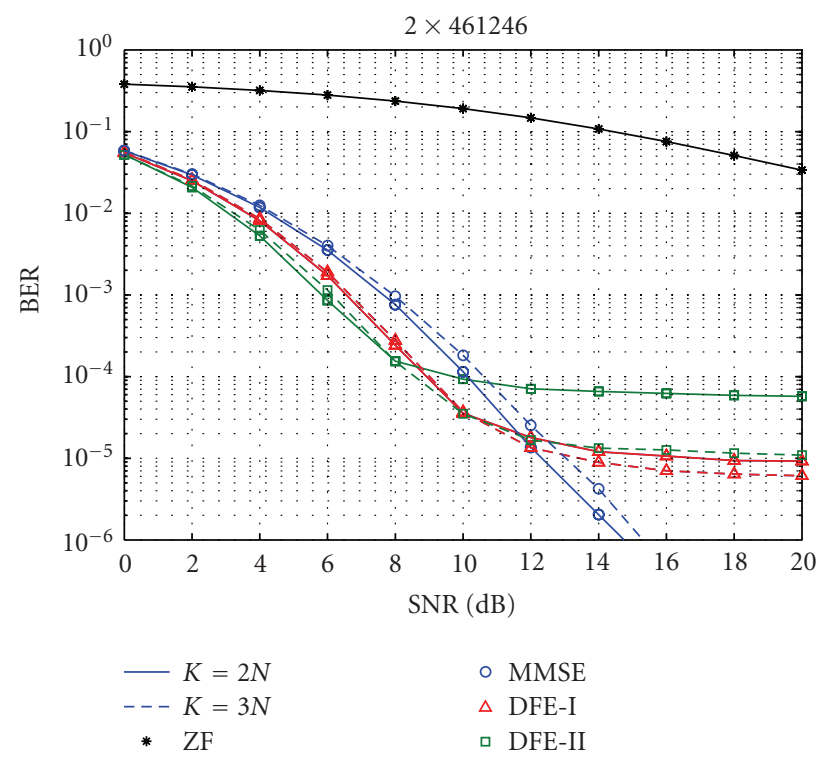

FIgURE 9: BER versus SNR for $N_{t} \times N_{r}=2 \times 4$ channel setup-II. time-varying basis functions $Q^{\prime \prime}=6$. For $K=N$, the BER curves of all equalizers suffer from an early error floor at a $\mathrm{BER}$ around BER $=3 \times 10^{-2}$. DFE-II marginally outperforms the linear MMSE equalizer and DFE-I. For $K=2 \mathrm{~N}$ and benchmarking at BER $=5 \times 10^{-3}$, an SNR gain of $4 \mathrm{~dB}$ and $5.8 \mathrm{~dB}$ are observed for DFE-I and DFE-II, respectively, compared to the linear MMSE equalizer.

5.2. Channel Setup-II. In this setup the number of basis functions is $Q=2$ for $K=2 N$ and $Q=4$ for $K=3 N$. An MIMO setup with $N_{t} \times N_{r}=2 \times 4$ is considered. The Linear MMSE and ZF equalizers as well as the nonlinear DFEs are evaluated. The feed-forward filters are designed such that $L^{\prime}=12$, and $Q^{\prime}=6$. The feedback filters are designed such that $L^{\prime \prime}=6$ and $Q^{\prime \prime}=4$. The simulation results corresponding to this set up are shown in Figure 9. For $K=2 N$, the BER curves for DFE-I and DFE-II show an error floor at BER $=9.2 \times 10^{-6}$ and $\mathrm{BER}=5.7 \times$ $10^{-5}$, respectively. Benchmarking at $\mathrm{BER}=10^{-4}$, DFE-I and DFE-II outperform the linear MMSE and ZF equalizers. An SNR gain of $1.3 \mathrm{~dB}$ and $2 \mathrm{~dB}$ are observed for DFE-I and DFE-II over the linear MMSE equalizer, respectively. The performance of the linear ZF equalizer is very marginal. For $K=3 N$, the different equalizers (the $\mathrm{ZF}$ does not exist for this case) exhibit almost the same performance as the $K=2 N$ case. A slight performance degradation is observed for the linear MMSE equalizer, this is due to the fact that for this case more parameters to equalize than the $K=2 \mathrm{~N}$ case. The DFE equalizers exhibit lower error floor. An error floor of BER $=6 \times 10^{-6}$ is observed for DFE-I and error floor at $\mathrm{BER}=10^{-5}$ for DFE-II. Again the BER floor of DFE-II is still higher than the error floor of DFE-I, which again confirms the fact that DFE-II is more vulnerable to error propagation than DFE-I even though with the modeling error is smaller due to the higher BEM resolution.

\section{Conclusions}

In this paper, time-varying FIR equalization techniques have been proposed for spatial multiplexing-based MIMO transmission over doubly selective channels. The time-varying FIR equalizers are designed considering the matched filter, MMSE and ZF criterion for linear and nonlinear decision feedback equalizers. The BEM is used to approximate the doubly selective channel, and to model and design the timevarying FIR feed-forward and feedback filters. By doing so, the one-dimensional time-varying deconvolution problem is reduced to a two-dimensional time-invariant deconvolution problem in the time-invariant coefficients of the channel BEM coefficients, and the time-invariant coefficients of the BEM equalizers. Using the BEM, and for a sufficient number of BEM parameters, the ZF solution exists for $N_{r} \geq N_{t}+1$, which extends a well-known result for the time-invariant MIMO equalization of frequency-selective channels. Using the extra degrees of freedom offered by the MIMO system, a DFE that feeds back the previously estimated symbols on all data streams to cancel/reduce ISI on a particular data stream can be obtained, which is shown to outperform the linear MMSE equalizer and the DFE that feeds back only the previously estimated symbols from one particular data stream. Block equalizers can be derived, but in general require high computational complexity. Hence, time-varying FIR equalization allows for lower complexity equalization techniques.

\section{References}

[1] G. J. Foschini and M. J. Gans, "On limits of wireless communications in a fading environment when using multiple antennas," Wireless Personal Communications, vol. 6, no. 3, pp. 311-335, 1998. 
[2] E. Telatar, "Capacity of multi-antenna Gaussian channels," European Transactions on Telecommunications, vol. 10, no. 6, pp. 585-595, 1999.

[3] L. Lindbom, M. Sternad, and A. Ahlén, "Tracking of timevarying mobile radio channels_part I: the Wiener LMS algorithm," IEEE Transactions on Communications, vol. 49, no. 12, pp. 2207-2217, 2001.

[4] E. Eweda, "Comparison of RLS, LMS, and sign algorithms for tracking randomly time-varying channels," IEEE Transactions on Signal Processing, vol. 42, no. 11, pp. 2937-2944, 1994.

[5] D. K. Borah and B. D. Hart, "A robust receiver structure for time-varying, frequency-flat, Rayleigh fading channels," IEEE Transactions on Communications, vol. 47, no. 3, pp. 360-364, 1999.

[6] D. K. Borah and B. D. Hart, "Receiver structures for timevarying frequency-selective fading channels," IEEE Journal on Selected Areas in Communications, vol. 17, no. 11, pp. 1863$1875,1999$.

[7] I. Barhumi, G. Leus, and M. Moonen, "Time-varying FIR equalization for doubly selective channels," IEEE Transactions on Wireless Communications, vol. 4, no. 1, pp. 202-214, 2005.

[8] I. Barhumi, G. Leus, and M. Moonen, "Time-varying FIR decision feedback equalization of doubly-selective channels," in Proceedings of the IEEE Global Telecommunications Conference (GLOBECOM '03), vol. 4, pp. 2263-2268, December 2003.

[9] A. Stamoulis, S. N. Diggavi, and N. Al-Dhahir, "Intercarrier interference in MIMO OFDM," IEEE Transactions on Signal Processing, vol. 50, no. 10, pp. 2451-2464, 2002.

[10] J. He, G. Gu, and Z. Wu, "MMSE interference suppression in MIMO frequency selective and time-varying fading channels," IEEE Transactions on Signal Processing, vol. 56, no. 8, pp. 36383651, 2008.

[11] A. P. Kannu and P. Schniter, "Minimum mean-squared error pilot-aided transmission for MIMO doubly selective channels," in Proceedings of the 40th Annual Conference on Information Sciences and Systems (CISS '06), pp. 134-139, Princeton, NJ, USA, March 2006.

[12] L. Yang, X. Ma, and G. B. Giannakis, "Optimal training for MIMO fading channels with time- and frequency-selectivity," in Proceedings of the IEEE International Conference on Acoustics, Speech, and Signal Processing (ICASSP '04), vol. 3, pp. III821-III824, Quebec, Canada, May 2004.

[13] J. K. Tugnait, S. He, and H. Kim, "Doubly selective channel estimation using exponential basis models and subblock tracking," IEEE Transactions on Signal Processing, vol. 58, pp. 1275-1289, 2010.

[14] S. M. Alamouti, "A simple transmit diversity technique for wireless communications," IEEE Journal on Selected Areas in Communications, vol. 16, no. 8, pp. 1451-1458, 1998.

[15] G. B. Giannakis and C. Tepedelenlioğlu, "Basis expansion models and diversity techniques for blind identification and equalization of time-varying channels," Proceedings of the IEEE, vol. 86, no. 10, pp. 1969-1986, 1998.

[16] G. Leus, S. Zhou, and G. B. Giannakis, "Orthogonal multiple access over time- and frequency-selective channels," IEEE Transactions on Information Theory, vol. 49, no. 8, pp. 19421950, 2003.

[17] X. Ma and G. B. Giannakis, "Maximum-diversity transmissions over doubly selective wireless channels," IEEE Transactions on Information Theory, vol. 49, no. 7, pp. 1832-1840, 2003. 